\title{
Inserção da fitoterapia em unidades de saúde da familia de São Luís, Maranhão: realidade, desafios e estratégias
}

\author{
Insertion of phytotherapy in family health units in São Luís, Maranhão state: reality, challenges and strategies
}

\section{La inserción de la fitoterapia en unidades de salud familiar de São Luís, Maranhão: realidad, desafíos y estrategias}

Waleska Regina Machado Araujo. Universidade Federal do Maranhão (UFMA). São Luís, MA, Brasil. waleska_regina@hotmail.com (Autora correspondente)

Rômulo Vieira Silva. Universidade Federal do Maranhão (UFMA). São Luís, MA, Brasil. vs_romulo@yahoo.com.br

Clemilson da Silva Barros. Universidade Federal do Maranhão (UFMA). São Luís, MA, Brasil. clemilson_2@yahoo.com.br

Flavia Maria Mendonça do Amaral. Universidade Federal do Maranhão (UFMA). São Luís, MA, Brasil. fmman@terra.com.br

\section{Resumo}

Objetivo: analisar a inserção da fitoterapia em unidades de saúde da família (USF) no município de São Luís, Maranhão, Brasil. Métodos: estudo analítico, observacional e transversal, tendo sido empregado como instrumento de coleta de dados um questionário semiestruturado com perguntas diretas, fechadas e abertas, aplicado aos diretores de USF do município. As variáveis da pesquisa foram categoria profissional, implantação de serviçoS de fitoterapia nas USF, benefícios da terapêutica na atenção primária, capacitação na área e desafios para efetivação do serviço, com análise quantitativa e qualitativa. Resultados: foram entrevistados 18 diretores, predominando os enfermeiros (50\%); 81\% dos entrevistados desconheciam as normativas vigentes relacionadas à fitoterapia no âmbito das políticas nacionais de saúde; $94 \%$ acreditavam que a oferta da fitoterapia como terapia alternativa e/ ou complementar traria benefícios à qualidade de vida da comunidade, sendo constatado ainda que $94 \%$ dos profissionais não tinham capacitação na área. Foram relatadas experiências pontuais referentes à fitoterapia por alguns diretores das unidades. Dentre os desafios citados pelos entrevistados, foi enfatizada a necessidade de estrutura física adequada e capacitação profissional. Conclusão: 0 estudo indica a real possibilidade da inserção da fitoterapia em USF mediante a promoção de mecanismos de capacitação profissional e educação permanente, a priorização do estudo de espécies vegetais locais de uso popular regional e a atuação efetiva da equipe multiprofissional, visando a qualificar a atenção primária e a ampliar o acesso às práticas alternativas e/ou complementares.

\section{Abstract}

Objective: to analyse the insertion of phytotherapy in family health units (FHU) in the municipality of São Luís, Maranhão state, Brasil. Methods: analytical, observational and cross-sectional study using a semi structured questionnaire with open and closed questions, applied to directors of FHU. The research variables were: professional category; implementation of phytotherapy in FHU; benefits of this therapy in primary care; professional continuing education/ training in phytotherapy; and challenges to effective services. Results: from the 18 directors interviewed we found that: $50 \%$ were nurses; $81 \%$ were unaware of the current regulations on phytotherapy within the national health policy; $94 \%$ believed that offering phytotherapy as alternative and complementary therapy would benefit the community's quality of life; and $94 \%$ of professionals did not have any training in phytotherapy. Personal experiences regarding phytotherapy were occasionally reported by the directors. Among the challenges mentioned by the respondents, the need for adequate physical structure and professional training was emphasized. Conclusion: the study indicates the real possibility of insertion of phytotherapy in FHU by promoting mechanisms for professional training and continuing education; studying regional plant species used by the local community; and the effective support of multidisciplinary team in order to qualify and expand primary care access to alternative and/or complementary practices.
Palavras-chave:

Fitoterapia

Estratégia Saúde da Família

Capacitação Profissional
Keywords:

Phytotherapy

Family Health Strategy

Professional Training 


\section{Resumen}

Objetivo: analizar la inserción de la fitoterapia en unidades de salud familiar (USF) en el municipio de São Luís, Maranhão, Brasil. Métodos: estudio analítico, observacional y transversal, siendo empleado un cuestionario semiestructurado, con preguntas directas, abiertas y cerradas, aplicado a los directores de USF en el municipio. Las variables de investigación fueron la categoría profesional; la inserción de servicios de fitoterapia en las USF; los beneficios de este tratamiento en la atención primaria; la capacitación en el área y los desafíos para un servicio efectivo. Resultados: fueran encuestados 18 directores, principalmente enfermeros (50\%); 81\% desconocían las normas vigentes relativas a la fitoterapia en la política nacional de salud; $94 \%$ creían que la oferta de la fitoterapia como terapia alternativa y complementaria beneficiaria la calidad de vida de la comunidad; y $94 \%$ de los profesionales no tenían capacitación en el área. Experiencias específicas relativas a la fitoterapia han sido reportadas por algunos directores de las unidades. Entre los desafíos citados por los encuestados se destacan la estructura física y la capacitación profesional adecuada. Conclusión: el estudio indica la real posibilidad de inserción de la fitoterapia en las USF mediante la promoción de mecanismos para capacitación profesional y educación continua; los estudios de especies de plantas locales de uso popular; y soporte de equipo multidisciplinario, con el fin de calificar la atención primaria y ampliar el acceso a las prácticas alternativas y/o complementarias.
Palabras clave:

Fitoterapia

Estrategia de Salud Familiar

Capacitación Profesional

\section{Introdução}

As práticas integrativas e complementares (PICs) no Brasil passaram a ser objeto de interesse governamental, de tal modo que recentemente o Ministério da Saúde (MS) implantou regulamentaçôes de estímulo à difusão da medicina complementar nos serviços do Sistema Único de Saúde (SUS). Partindo-se da perspectiva de que as políticas de saúde materializam-se na ponta do sistema, por meio de ações dos diversos atores sociais, merece atenção o surgimento de novas práticas de saúde distintas da racionalidade médica ocidental. ${ }^{1,2,3}$

$\mathrm{Na}$ ampliação das ofertas terapêuticas do SUS e no atendimento aos seus princípios de base, em 2006, foi lançada a Política Nacional de Práticas Integrativas e Complementares no Sistema Único de Saúde para estimular sistemas e recursos terapêuticos que envolvam mecanismos naturais de prevenção de agravos e recuperação da saúde por meio de tecnologias eficazes e seguras. ${ }^{4}$ No Brasil, a Atenção Primária à Saúde (APS) é prioridade, e a Estratégia Saúde da Família (ESF) é fundamental para o fortalecimento das açôes de atenção primária no SUS, sendo considerada como a ordenadora das redes de atenção e coordenadora do cuidado com foco na família e na comunidade. ${ }^{5}$ A inclusão das PICs na ESF, portanto, está de acordo com os princípios de universalidade, integralidade e equidade que estruturam o SUS.

A fitoterapia é caracterizada pelo uso de plantas medicinais em suas diferentes formas farmacêuticas, sem a utilização de substâncias ativas isoladas. ${ }^{4}$ As iniciativas para formulação de políticas e programas com ênfase nessa área atenderam às diretrizes e recomendações de várias conferências e da Organização Mundial de Saúde (OMS). Ações decorrentes dessas políticas culminaram, em 2006, no Programa Nacional de Plantas Medicinais e Fitoterápicos (PNPMF) agregando açôes, gestores, órgãos envolvidos, prazos e origem dos recursos para implantação, com abrangência em toda a cadeia produtiva de fitoterápicos. ${ }^{6}$ Nesse sentido, é fundamental a análise da inserção da fitoterapia nas políticas de saúde no país, estados e municípios. Este estudo pretende analisar a inserção da fitoterapia em Unidades de Saúde da Família (USF) no município de São Luís, estado do Maranhão, Brasil, na visão dos diretores de USF, visando a conhecer os desafios e a propor estratégias para sua implantação.

\section{Métodos}

Estudo analítico, observacional e transversal, tendo sido empregado como instrumento de coleta de dados um questionário semiestruturado. Foram utilizadas perguntas diretas, fechadas e abertas, tendo como variáveis categoria profissional, implantação de serviços de fitoterapia nas USF, benefícios dessa terapêutica na atenção primária, capacitação na área e desafios para efetivação do serviço.

Considerando-se que grande parte da cobertura da atenção primária no Brasil é realizada mediante a ESF, a pesquisa foi realizada em USF do município de Sáo Luís, capital do estado do Maranhão, durante o mês de novembro de 2011. São Luís tem uma população de 1.014.837 habitantes, com rede de saúde composta por 81 unidades de saúde, distribuídas em sete distritos sanitários, das quais 35 são USF.7

Neste estudo, optou-se por realizar a entrevista com diretores dessas USF por se entender que a gerência dos serviços proporciona um protagonismo nas açôes de saúde, sendo uma ferramenta importante na efetivação de políticas públicas. ${ }^{8}$ Assim, a amostra deste estudo foi constituída por diretores de 24 USF, sendo excluídas as USF de um distrito sanitário, dada a dificuldade de acesso. 
O acesso às USF foi facultado pela Secretaria Municipal de Saúde (SMS). Após prévio agendamento, os sujeitos que aceitaram participar da pesquisa assinaram o Termo de Consentimento Livre e Esclarecido (TCLE), totalizando 18 diretores. As USF foram identificadas por letras do alfabeto (USF A - USF R), e foi preservada a identidade dos participantes.

Após a coleta de dados, os mesmos foram sistematizados, organizados e analisados, sendo comparados à literatura, possibilitando assim apresentarem-se estratégias de implantação da fitoterapia na atenção primária do município com base nos princípios de intersetorialidade e equipe multiprofissional. O projeto de pesquisa foi aprovado pelo Comitê de Ética em Pesquisa do Hospital Universitário Presidente Dutra (HUPD), com parecer no 348/11, de acordo com a Resoluçáo no 196/96 do Conselho Nacional de Saúde.

\section{Resultados e discussão}

Os participantes da pesquisa foram distribuídos em sete categorias profissionais, compostos por enfermeiros (9); gestores hospitalares (2); gestores de saúde (2); cirurgiōes-dentista (2); farmacêutico (1); assistente social (1) e administrador (1). Constatou-se o predomínio de enfermeiros (50\%), o que pode ser explicado pelo início da implantação do Programa Saúde da Família (PSF) em 1994, quando a formação da equipe multiprofissional era composta basicamente por, no mínimo, um médico, um enfermeiro, um auxiliar ou técnico de enfermagem e agentes comunitários de saúde (ACS). ${ }^{9}$ Vale enfatizar ainda que disciplinas com foco no gerenciamento têm sido incorporadas historicamente na estrutura curricular dos cursos de graduação em enfermagem, o que contribui para o desenvolvimento de competências e habilidades gerenciais como a atenção à saúde, a tomada de decisões, a comunicação, a liderança e a administração de unidades de saúde. ${ }^{10,11}$

A ausência dos médicos demonstra a dificuldade para atrair essa categoria ao campo da saúde pública podendo ser associada à fragilidade na formação desse profissional para sistematização e implementação do SUS e também pela grande oferta de outras oportunidades, o que favorece a possibilidade de se obter nível social mais alto e com rendimentos melhores. ${ }^{12,13} \mathrm{~A}$ baixa presença dos cirurgiōes-dentistas pode ser explicada pela inserção mais recente da saúde bucal na Estratégia Saúde da Família. ${ }^{12}$ Com a criação do Núcleo de Apoio à Saúde da Família (NASF), houve uma ampliação de categorias profissionais que poderiam apoiar as práticas em saúde nos territórios sob responsabilidade das equipes de saúde da família com o objetivo de ampliar a abrangência e resolutividade da ESF. ${ }^{14}$

Quando indagados quanto às políticas públicas para a implantação da fitoterapia, $81 \%$ dos entrevistados desconheciam as normativas vigentes relacionadas à fitoterapia no âmbito das políticas nacionais de saúde e $56 \%$ não conheciam o PNPMF. Esse resultado está em concordância com o de outras localidades, já que estudos nacionais demonstram que a maioria dos profissionais desconhece as diretrizes nacionais, ${ }^{15-17}$ situaçáo preocupante considerando-se que esse representa o principal instrumento para orientação dos gestores na implantação desses serviços. ${ }^{4}$ Ischkanian $^{18}$ atribui o desconhecimento à ineficiência dos meios de divulgação dessas políticas para as unidades de saúde devido à preferência dos profissionais pelo modelo biomédico, à descrença dos profissionais decorrente da própria formação acadêmica e aos preconceitos que cercam o tema. Apenas o diretor de uma unidade referiu que alguns profissionais vêm orientando, esporadicamente, o uso de fitoterápicos, conforme depoimento:

"Alguns profissionais já orientam o uso de medicamentos fitoterápicos, alguns prescrevem e indicam locais onde a pessoa pode encontrar" (USF G).

Entende-se que essa realidade está relacionada à falta de profissionais que assumam a prescrição de fitoterápicos dentro da USF, com orientaçóes para o uso dos mesmos na comunidade, bem como à falta de acesso e disponibilidade de tal recurso terapêutico. Estudo realizado em Unidades de Saúde no Ceará, estado pioneiro em assistência fitoterápica, indica o registro de 20,6\% das prescriçóes de pacientes com idade igual ou superior a 18 anos contendo pelo menos 01 medicamento fitoterápico, refletindo a realidade do Programa Farmácia Viva no Estado, com produção e dispensação de medicamentos fitoterápicos mediante prescrição do profissional de saúde ${ }^{19}$ e em atendimento a determinaçóes técnicas que instituem as Farmácias Vivas no âmbito do SUS. ${ }^{20}$ No presente estudo, em apenas $28 \%$ das USF houve referência de iniciativa anterior da produçáo de plantas com fins medicinais, mas, segundo os gestores, a descontinuidade da mesma foi ocasionada por falta de manutenção. Foram relatadas experiências pontuais, por iniciativa do diretor ou de um profissional lotado na unidade, sendo as mesmas descontinuadas por falta de apoio, conforme depoimento a seguir:

"Participei de uma Conferência Ambiental, onde ensinaram algumas técnicas de cultivo... resolvi, com ajuda de algumas pessoas da comunidade, fazer canteiros de plantas no espaço lateral dentro da unidade. Mas as plantas acabaram morrendo por falta de manutençẫo e cuidados no solo." (USF Q) 
"Tive conhecimento no ano passado, através de uma médica da unidade, que incentivou a plantação de algumas plantas medicinais, inclusive horta.” (USF D)

"Há alguns anos atrás, em outra gestão, houve um início de projeto, mas não foi adiante" (USF A).

Dados do Ministério da Saúde indicam que em 25\% dos programas de fitoterapia a implantação foi feita com recursos estaduais ou federais, mas a sua manutenção acontece com recursos próprios, demonstrando assim que os programas implantados e mantidos até então o foram em decorrência da sensibilização de gestores. Tal fato constitui um fator de instabilidade para o andamento desses programas, uma vez que os mesmos iriam depender da vontade política do gestor de implantar a fitoterapia no seu estado ou município ${ }^{4}$. Estudo demonstra que municípios que chegaram a implantar esses serviços, mas que depois o abandonaram, justificaram a náo continuidade do projeto pela falta de incentivo dos gestores, falta de divulgação junto aos usuários e, consequentemente, baixa demanda por parte da populaçáo. ${ }^{21}$

$\mathrm{Na}$ avaliação da percepção dos diretores das USF quanto à inserção da fitoterapia como estratégia na atenção primária, 94\% acreditavam que isso traria benefícios à comunidade, sendo mais uma opção na busca de promoção da saúde, podendo reduzir custos com medicamentos. A crescente ascensão da fitoterapia na sociedade contemporânea como alternativa e/ou complemento terapêutico tem motivado diversos estudos que comprovaram seus benefícios na atenção primária, aliados à busca da qualidade da atenção, à validação do conhecimento popular/tradicional, ao estreitamento de laços da comunidade com a equipe de saúde, ao fortalecimento do controle social, à integralidade em saúde mediante a ampliação do olhar e das ofertas de cuidado, assim como ao vínculo e respeito pelos valores culturais. ${ }^{1522-24}$

Apesar da constatação da inexistência da implantação da fitoterapia nas USF selecionadas neste estudo, 83\% dos entrevistados referiram que a comunidade local atendida recorre às práticas alternativas de saúde, relatando que alguns usuários fazem o uso em suas residências de chás, lambedores (xaropes caseiros) e outras apresentaçóes à base de plantas medicinais. Tal situação reflete um comportamento semelhante ao de diversas regióes do país onde a comunidade afirma fazer uso de espécies vegetais in natura na forma de chás, preparados por decocção ou infusão, macerados ou formas de uso externo, além de adquirir plantas secas, produto fitoterápico manipulado e fitoterápico industrializado. ${ }^{24-26}$ Indiscutivelmente, o uso seguro e racional de plantas medicinais tem papel fundamental em diversas comunidades, sendo prioritário na atenção a saúde. Entretanto, deve-se primar pela qualidade do material vegetal utilizado, evitando exposição a riscos por aquisição de produto impróprio para o consumo. ${ }^{27,28}$

Quando questionados sobre os principais desafios para implantação do programa de fitoterapia, os entrevistados destacaram: o compromisso do gestor principal; a necessidade de parcerias com centros de ensino e pesquisa; a aceitaçáo dos prescritores; a disponibilidade de recursos humanos; a regulamentação para o serviço; o incentivo à comunidade; o apoio da Secretaria Municipal de Saúde; a capacitaçáo dos profissionais; e a estrutura adequada na unidade (Figura 1).

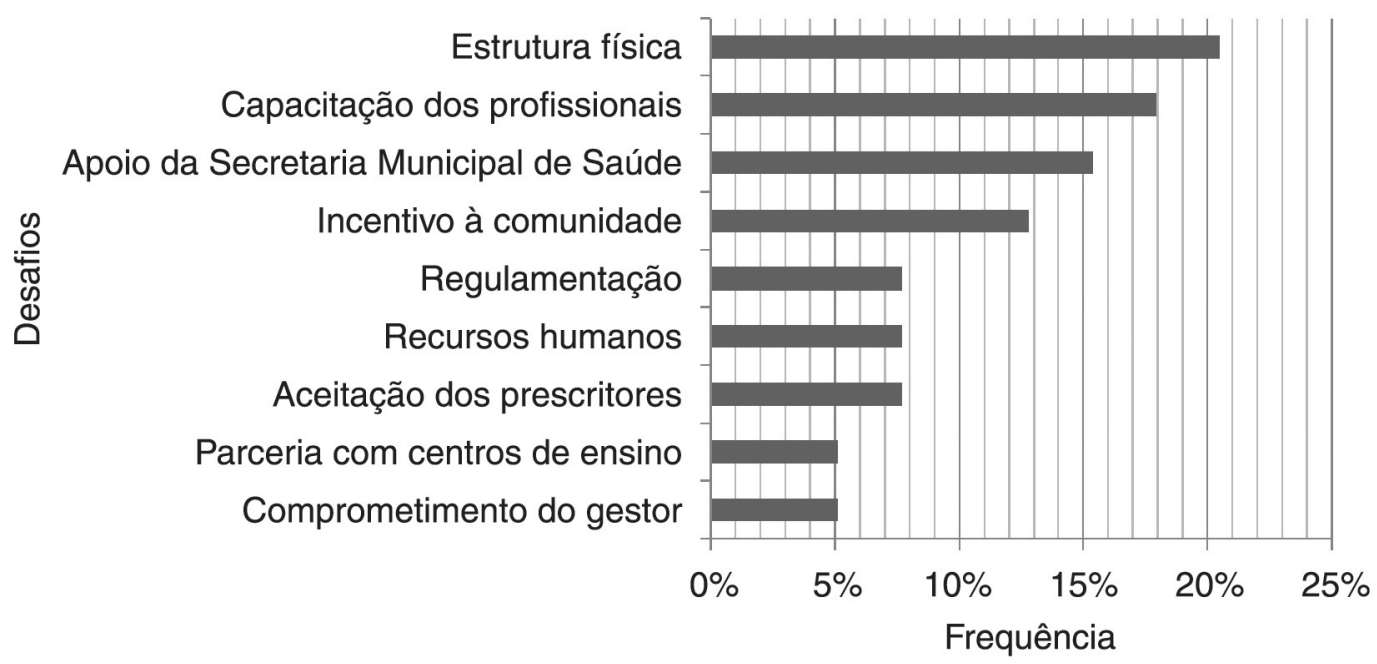

Figura 1. Frequência de desafios para a implantação do programa de fitoterapia referidos pelos diretores das unidades de saúde da família do município de São Luís, Maranhão, 2011. 
Observa-se que a estrutura física da unidade de saúde e a capacitação profissional foram os desafios mais referidos pelos diretores (21\% e 18\%, respectivamente). Em diagnóstico de programas de fitoterapia em outras regiôes, foram apresentadas como principais dificuldades: a formação acadêmica deficiente; a sensibilização dos gestores; o apoio institucional e político; e a infraestrutura para se garantirem serviços com qualidade, segurança e eficácia. ${ }^{15,29}$

Neste estudo, foi verificado que $94 \%$ dos entrevistados não tinham capacitação na área de práticas alternativas de saúde. A capacitação de profissionais de saúde aparece em muitos estudos como desafio indiscutível para a institucionalização da fitoterapia no SUS, ${ }^{15,22,23,30}$ incluindo capacitaçáo dos prescritores, ${ }^{16}$ passando a ser uma necessidade de saúde pública.

Os profissionais da equipe de saúde, principalmente os agentes comunitários de saúde, que agem diretamente nas casas das famílias, são importantes como recursos de educação em saúde, pois atuam como multiplicadores de saúde ao dialogarem com os saberes populares e saberes médicos-científicos em saúde. ${ }^{31}$ Os desafios para a implantação de um programa de fitoterapia identificados neste estudo foram semelhantes aos dados encontrados em outros estudos. ${ }^{18,32}$ Diante do exposto, torna-se evidente que a institucionalização da fitoterapia deve ser acompanhada de políticas de incentivo e de infraestrutura adequadas para funcionamento do serviço, com padróes de qualidade em toda a cadeia produtiva de plantas medicinais e fitoterápicas.

Com base nos resultados do presente estudo e na literatura da área, é possível propor estratégias na perspectiva real de inserção da fitoterapia como prática racional tais como: a oferta da disciplina fitoterapia nos cursos de graduação da área de saúde, bem como cursos lato sensu e stricto sensu em fitoterapia; inserção da assistência fitoterápica na ESF com apoio da equipe multiprofissional; programa de sensibilização a gestores, profissionais de saúde e movimentos populares; implantação e estruturação da Farmácia Viva conforme determinaçóes técnicas vigentes; estímulo a estudos da fitogeografia da regiāo; criação de hortos medicinais com cultivo controlado bem como um banco de dados de plantas medicinais e fitoterápicos para consulta pela equipe de saúde.

\section{Conclusão}

De acordo com o presente estudo, há uma inexistência de oferta de serviços de fitoterapia nas USF do município de São Luís, Maranháo. A maioria dos diretores entrevistados desconhecia as políticas e normas vigentes, apesar de referirem a crença nos benefícios da terapêutica à qualidade de vida das pessoas e, ainda, reconhecerem que a comunidade local refere o uso popular de plantas como recurso terapêutico.

Os desafios mais referidos para a implantaçáo da fitoterapia na atenção primária foram a estrutura física das unidades e a capacitação profissional. Sendo assim, é possível definir estratégias para qualificar a atenção primária e ampliar o acesso à fitoterapia como prática alternativa e complementar. Para tal, sabe-se que quaisquer propostas somente terão êxito caso haja o reconhecimento e o compromisso na política governamental para a real implantação na atenção primária, o que envolve mudanças na percepçáo do processo saúde-doença no contexto social e cultural.

\section{Referências}

1. Pinheiro R, Mattos R, Camargo Jr KR. Construção da integralidade: cotidiano, saberes e práticas em saúde. $4^{a}$ ed. Rio de Janeiro: ABRASCO; 2007.

2. Minayo MCS, Coimbra Jr CEA. Críticas e atuantes: ciências sociais e humanas em saúde na América Latina. Rio de Janeiro: Fiocruz; 2005.

3. Andrade JT, Costa LFA. Medicina complementar no SUS: práticas integrativas sob a luz da Antropologia médica. Saúde Soc. 2010;19:497-508. http://dx.doi.org/10.1590/S0104-12902010000300003.

4. Ministério da Saúde (BR), Secretaria de Atenção à Saúde, Departamento de Atenção Básica. Política Nacional de Práticas Integrativas e Complementares no SUS - PNPIC-SUS. Brasília: MS; 2006.

5. Pereira AMM, Castro ALB, Oviedo RAM, Barbosa LG, Gerassi CD, Giovanella L. Atenção primária à saúde na América do Sul em perspectiva comparada: mudanças e tendências. Saúde Debate. 2012;36(94):482-99. http://dx.doi.org/10.1590/S0103-11042012000300019.

6. Ministério da Saúde (BR), Secretaria de Ciência, Tecnologia e Insumos Estratégicos, Departamento de Assistência Farmacêutica e Insumos Estratégicos. Programa Nacional de Plantas Medicinais e Fitoterápicos. Brasília: MS; 2009.

7. Prefeitura de São Luís, Secretaria Municipal de Saúde. Guia de distritos. 2013. [acesso em 2013 Set 16]. Disponível em: http://www.saoluis.ma.gov.br/SEMUS. 
8. Vanderlei MIG, Almeida MCP. A concepção e prática dos gestores e gerentes da estratégia de saúde da família. Ciênc Saúde Coletiva. 2007;12(2):443-53. http://dx.doi.org/10.1590/S1413-81232007000200021.

9. Ministério da Saúde (BR). Portaria nº 1886 de 18 de dezembro de 1997. Aprova as Normas e Diretrizes do Programa de Agentes Comunitários de Saúde e do Programa de Saúde da Família. Diário Oficial da União. 1997 Dec 22; Seção 1. Portuguese.

10. Rothbarth S, Wolff LDG, Peres AM. O desenvolvimento de competências gerenciais do enfermeiro na perspectiva de docentes de disciplinas de administração aplicada à enfermagem. Texto Contexto Enferm. 2009;18(2):321-29. http://dx.doi.org/10.1590/S0104-07072009000200016.

11. Cunha I, FRG XN. Competências gerenciais de enfermeiras: um novo velho desafio. Texto Contexto Enferm. 2006;15(3):479-82. http://dx.doi.org/10.1590/S0104-07072006000300013.

12. Gil CRR. Formação de recursos humanos em saúde da família: paradoxos e perspectivas. Cad Saúde Pública. 2005;21(2):490-98. http://dx.doi.org/10.1590/S0102-311X2005000200015.

13. Medeiros CRG, Junqueira A, Schwingel G, Carreno I, Jungles LAP, Saldanha O. A rotatividade de enfermeiros e médicos: um impasse na implementação da Estratégia de Saúde da Família. Ciênc Saúde Coletiva. 2007;15(1):1521-31. http://dx.doi.org/10.1590/S1413-81232010000700064.

14. Ministério da Saúde (BR). Portaria nº 154 de 24 de janeiro de 2008. Cria os Núcleos de Apoio à Saúde da Família - NASF. Brasil: MS; 2008.

15. Fontenele RP, Sousa DMPd, Carvalho ALM, Oliveira FdA. Fitoterapia na Atenção Básica: olhares dos gestores e profissionais da Estratégia Saúde da Família de Teresina (PI), Brasil. Ciênc Saúde Coletiva. 2013;18(8):2385-94. http://dx.doi.org/10.1590/S1413-81232013000800023.

16. Cantarelli AP. Estudo da utilização de plantas medicinais pelos usuários do SUS e das práticas dos profissionais de saúde de Doutor Maurício Cardoso em relação à fitoterapia [Monografia]. Porto Alegre: Curso de Especialização em Gestão em Saúde EAD. Escola de Administração, Universidade Federal do Rio Grande do Sul; 2012. Portuguese. http://hdl.handle.net/10183/67671.

17. Thiago SCS, Tesser CD. Percepção de médicos e enfermeiros da Estratégia de Saúde da Família sobre terapias complementares. Rev Saúde Pública. 2011;45(2):249-57. http://dx.doi.org/10.1590/S0034-89102011005000002.

18. Ischkanian PC, Pelicioni MCF. Challenges of complementary and alternative medicine in the SUS aiming to health promotion. Journal of Human Growth and Development. 2012;22(2):233-38.

19. Silva MIG, Gondim APS, Nunes IFS, Sousa FCF. Utilização de fitoterápicos nas unidades básicas de atenção à saúde da família no município de Maracanaú (CE). Rev Bras Farmacogn. 2006;16(4):455-62. http://dx.doi.org/10.1590/S0102-695X2006000400003.

20. Ministério da Saúde (BR). Portaria no 886 de 20 de abril de 2010. Institui a Farmácia Viva no âmbito do Sistema Único de Saúde (SUS). Brasil: MS; 2010.

21. Camargo EES, Bandeira MAM, Oliveira AGD. Diagnosis of public programs focused on herbal medicines in Brazil. Bol Latinoam Caribe Plantas Med Aromát. 2012;11(4):362-368.

22. Sampaio LA, Oliveira DR, Kerntopf MR, Brito Jr FE, Menezes IRA. Percepção dos enfermeiros da estratégia saúde da família sobre o uso da fitoterapia. REME Rev Min Enferm. 2012;17(1):76-84. http://dx.doi.org/10.5935/1415-2762.20130007.

23. Rosa C, Câmara SG, Béria JU. Representações e intenção de uso da fitoterapia na atenção básica à saúde. Ciênc Saúde Coletiva. 2011;16(1):311-18. http://dx.doi.org/10.1590/S1413-81232011000100033.

24. Batista LM, Valença AMG. A fitoterapia no âmbito da Atenção Básica no SUS: realidades e perspectivas. Pesq Bras Odontoped Clin Integr. 2012;12(2):293-96.

25. Schwambach KH, Amador TA. Estudo da utilização de plantas medicinais e medicamentos em um município do sul do Brasil. Latin American Journal Pharmacy. 2007;26(4):602. http://hdl.handle.net/10915/7523.

26. Agra MF, Freitas PF, Barbosa-Filho JM. Synopsis of the plants known as medicinal and poisonous in Northeast of Brazil. Rev Bras Farmacogn. 2007;17(1):114-40. http://dx.doi.org/10.1590/S0102-695X2007000100021.

27. Amaral F, Coutinho D, Ribeiro M, Oliveira M. Avaliação da qualidade de drogas vegetais comercializadas em São Luís/Maranhão. Rev Bras Farmacogn. 2003;13:27-30. http://dx.doi.org/10.1590/S0102-695X2003000300011.

28. Badke MR, Budó MLD, Alvim NAT, Zanetti GD, Heisler EV. Saberes e práticas populares de cuidado em saúde com o uso de plantas medicinais. Texto Contexto Enferm. 2012;21(2):363-70. http://dx.doi.org/10.1590/S0104-07072012000200014.

29. Michiles E. Diagnóstico situacional dos serviços de fitoterapia no Estado do Rio de Janeiro. Rev Bras Farmacogn. 2004;14(1):16-19. http://dx.doi.org/10.1590/S0102-695X2004000300007.

30. Santos RL, Guimaraes GP, Nobre MSC, Portela AS. Análise sobre a fitoterapia como prática integrativa no Sistema Único de Saúde. Rev Bras Plantas Med 2011;13(4):486-91. http://dx.doi.org/10.1590/S1516-05722011000400014.

31. Nunes MO, Trad LB, Almeida BA, Homem CR, Melo MCC. O agente comunitário de saúde: construção da identidade desse personagem híbrido e polifônico. Cad Saúde Pública. 2002;18(6):1639-46. http://dx.doi.org/10.1590/S0102-311X2002000600018.

32. Silvello CLC. O uso de plantas medicinais e de fitoterápicos no SUS: uma revisão bibliográfica [Monografia]. Porto Alegre: Escola de Enfermagem, Universidade Federal do Rio Grande do Sul; 2010. Portuguese. http://hdl.handle.net/10183/28232. 